\title{
Metastatic Pancreatic Cancer: Current Treatment Options for Swiss Patients
}

\author{
Sara De Dosso ${ }^{1}$, Alexander R. Siebenhüner ${ }^{2}$, Thomas Winder ${ }^{3}$, Alexander Meisel' ${ }^{4}$, \\ Ralph Fritsch ${ }^{4}$, Christoforos Astaras ${ }^{5}$, Petr Szturz ${ }^{6}$, Markus Borner ${ }^{7}$
}

\section{ABSTRACT}

Despite oncological advances over the past years, survival outcomes for patients with pancreatic ductal adenocarcinoma (PDAC) remain poor. For most PDAC patients, the disease is often asymptomatic at early phases and is therefore typically diagnosed at the advanced or metastatic stage. As a result, up to $85 \%$ of the cases are unresectable, and systemic chemotherapy is the predominant treatment modality for this patient population. While the current therapeutic strategies for the local disease include surgical resection and adjuvant chemotherapy, FOLFIRINOX and gemcitabine plus nab-paclitaxel regimens are the frontline standard of care in the unresectable locally advanced and metastatic settings. In Switzerland, nanoliposomal irinotecan (nal-IRI) is currently the only regimen approved in the second line following progression on gemcitabine plus nab-paclitaxel, based on the results from the phase III NAPOLI-1 trial. Recently, olaparib, a poly (ADP-ribose) polymerase (PARP) inhibitor, and larotrectinib, a first-in-class tropomyosin receptor kinases (TRK) inhibitor, were added to the treatment armamentarium of patients with a molecularly-defined subset of metastatic adenocarcinoma of the pancreas. This article provides an overview of the current treatment landscape of pancreatic cancer in Switzerland. In addition, we report a case of a long-term survivor with advanced pancreatic adenocarcinoma who achieved a notably good response to nal-IRI plus 5-FU/LV after progression on gemcitabine plus nab-paclitaxel.

Keywords: pancreatic adenocarcinoma, FOLFIRINOX, gemcitabine, nab-paclitaxel, nanoliposomal irinotecan

\section{INTRODUCTION}

Pancreatic ductal adenocarcinoma (PDAC) is the most prevalent malignant disease of the pancreas, accounting for about $90 \%$ of all pancreatic cancer cases. ${ }^{1}$ PDAC is characterized by low incidence and a high mortality rate. Although it accounts for only $3 \%$ of all cancers, it is the seventh leading cause of cancer-related death, with a mortality rate of $98 \%{ }^{2,3}$ The highest incidence rates of PDAC have been reported in Europe, North America and Australia/ New Zealand (7-10 new cases/100,000 people/year). ${ }^{3-6}$ In Switzerland, the average annual PDAC incidence between 2012 and 2016 was 715 in men and 708 in women, with an average annual mortality of 615 and 643 , respectively. ${ }^{7,8}$ Despite some significant treatment advances over the past years, PDAC continues to have a poor prognosis, with a 5 -year survival rate of 5-10\% worldwide. ${ }^{9-11}$ In Switzerland, the 5-year survival rate was about 5\% in men and $6.8 \%$ in women between 2001 and $2010 .{ }^{11}$ The incidence and mortality rates correlate with increasing age, with the most prevalent patient group comprising patients above 70 years of age. ${ }^{5}$ Other factors that considerably impact the prognosis are disease stage at the time of diagnosis, tumor size and the presence of liver metastases. ${ }^{5,12}$

The most common modifiable lifestyle risk factors for developing pancreatic cancer are smoking, obesity, alcohol abuse and chronic pancreatitis, while non-modifiable risk factors include increased age, gender, race, diabetes mellitus, family history and genetic predisposition, Helicobacter pylori infection and blood group. ${ }^{5,11,13,14}$ Genetic predisposition, such as germline mutations in the ATM, BRCA1, BRCA2, CDKN2A, PALB2, PRSS1, STK11, TP53, and the Lynch syndrome (LS) mismatch repair genes, play a key role in about $10 \%$ of PDACs. ${ }^{14}$
${ }^{1}$ Oncology Institute of Southern Switzerland (IOSI), Bellinzona, Switzerland

${ }^{2}$ Cantonal Hospital Schaffhausen, Schaffhausen, Switzerland ${ }^{3}$ Swiss Tumor Molecular Institute, OnkoZentrum, Zurich, Switzerland ${ }^{4}$ University Hospital Zurich, Zurich, Switzerland

${ }_{5}^{5}$ Geneva University Hospitals (HUG), Geneva, Switzerland ${ }^{6}$ Lausanne University Hospital (CHUV), Lausanne, Switzerland 7 Oncocare, Engeriedspital, Engeried, Switzerland

Corresponding Author:

PD Dr Sara De Dosso

Oncology Institute of Southern

Switzerland (IOSI)

Bellinzona, Switzerland

Email: Sara.DeDosso@eoc.ch

Tel: +41 (0)918119302

Fax: +41 (0)918118734

DOI: $10.36000 / \mathrm{hbT} .0 \mathrm{OH} .2021 .08 .032$ ISSN: 2673-2092 (Print) and 26732106 (Online)

This article was received on May 07, 2021

This article was accepted on June 08, 2021

This article was published on June 11, 2021

De Dosso S et al. Metastatic Pancreatic Cancer: Current Treatment Options for Swiss Patients. healthbook TIMES Onco Hema 2021; (8): 42-49 


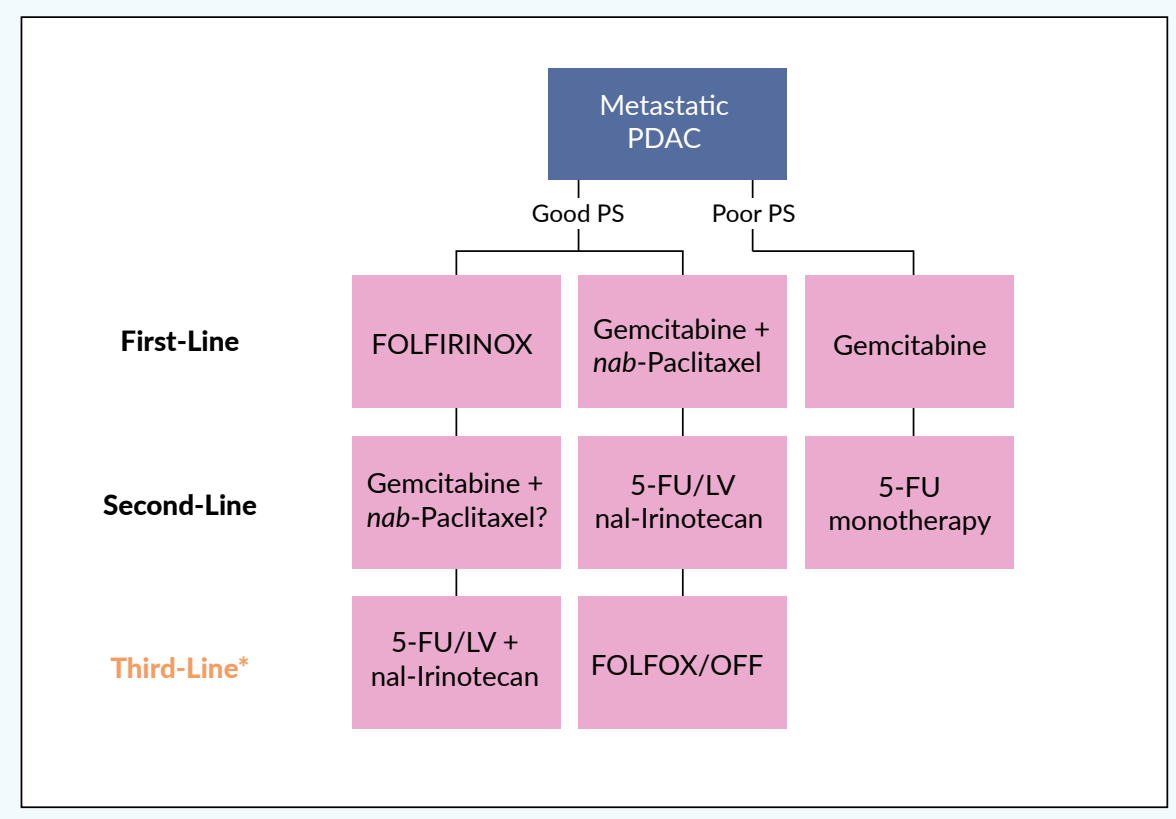

Figure 1. Treatment strategy for first-, second- and third-line therapy in patients with metastatic pancreatic ductal adenocarcinoma (PDAC). ${ }^{*}$ Clinical trials investigating targeted therapies according to the molecular profile should be preferred. PS, performance status. Adapted from De Dosso et al. 2021. ${ }^{23}$
Although early disease detection may be critical to reducing mortality, population-based screening is not considered effective for detecting early-stage PDAC as the lifetime risk of developing pancreatic cancer is considerably low (about $1 \%)$ in an unselected population. ${ }^{15}$ However, for screening of targeted groups like patients with a family history of this disease, new and more effective techniques are currently under development, including blood markers CA19-9, CA-50, SPAN-1, DUPAN-2, cell surface-associated mucins (MUC), carcinoembryonic antigen and heat shock proteins. ${ }^{5}$ In patients with LS and one first-degree relative affected with PDAC, annual magnetic resonance imaging (MRI) and/or endoscopic ultrasound surveillance may be considered. ${ }^{16}$

\section{CURRENT TREATMENT STRATEGIES FOR PANCREATIC CANCER}

The median survival for untreated PDAC patients with metastatic disease is less than 6 months. Patients often experience rapid symptomatic progression presenting with cachexia, anorexia, bile duct and gastric outlet obstruction as well as pain, underlining the importance of optimal palliative care. According to the ASCO Clinical Practice Guideline Update, a full assessment of symptom burden, psychological status and social support should be performed as early as possible, preferably at the first visit. ${ }^{17}$ This assessment will indicate a need for a formal palliative care consultation and services in most metastatic PDAC patients.

\section{FIRST-LINE PALLIATIVE SETTING}

For years, gemcitabine as monotherapy has been the only therapeutic option in patients with metastatic pancreatic adenocarcinoma. ${ }^{18-21}$ In Switzerland, both folinic acid (leucovorin $[\mathrm{LV}]$ ), fluorouracil (5-FU), irinotecan and oxaliplatin and gemcitabine plus nab-paclitaxel are the current standard of care in advanced PDAC patients in the first-line setting (Figure 1). In the phase III PRODIGE/ACCORD 11 trial, FOLFIRINOX (folinic acid, 5-FU, irinotecan, and oxaliplatin) demonstrated improved survival outcomes, as compared with gemcitabine. ${ }^{22}$ In this study, 342 treatmentnaïve patients with metastatic pancreatic cancer and an Eastern Cooperative Oncology Group (ECOG) performance status (PS) of 0 or 1 were randomized to receive either FOLFIRINOX or gemcitabine. At data cutoff, FOLFIRINOX was associated with both prolonged median overall survival (OS) (11.1 months vs 6.8 months with gemcitabine; HR: 0.57 [95\% CI: $0.45-0.73] ; \mathrm{p}<0.001)$ and median progression-free survival (PFS) (6.4 months vs 3.3 months; HR: 0.47 [95\% CI: $0.37-0.59] ; \mathrm{p}<0.001)$. The objective response rate (ORR) was $31.6 \%$ with FOLFIRINOX and $9.4 \%$ with gemcitabine $(\mathrm{p}<0.001)$. Regarding safety, more adverse events (AEs) were noted in the FOLFIRINOX group, with incidences of grade 3-4 neutropenia, febrile neutropenia, thrombocytopenia, diarrhea, and sensory neuropathy being significantly higher with FOLFIRINOX than gemcitabine. Due to its toxicity profile, FOLFIRINOX is suitable only in a highly selected population, primarily for the treatment of younger $(<65$ years old) and fit patients with normal liver function.

In Switzerland, the most frequently utilized first-line drug therapy for locally advanced unresectable or metastatic PDAC is gemcitabine plus nab-paclitaxel. ${ }^{23}$ The approval of this combination was based on the results from the phase III MPACT trial, which showed that gemcitabine plus nab-paclitaxel were associated with significantly improved long-term survival outcomes compared with gemcitabine alone. ${ }^{24,25}$ In the primary analysis of the trial, treatment with the doublet led to a significantly improved median OS 
versus gemcitabine alone (8.5 months vs 6.7 months, HR: 0.72 [95\% CI: 0.62-0.83]; p<0.001). ${ }^{24}$ Also, both PFS and overall response rate (ORR) were significantly improved. Although the combination regimen was slightly more toxic than monotherapy, AEs were managed by dose reductions and treatment delays. ${ }^{26,27}$ The long-term survival analysis with a median follow-up of 13.9 months confirmed that the median OS was significantly longer in the nab-paclitaxel plus gemcitabine versus the gemcitabine alone arm ( 8.7 months vs 6.6 months, HR: 0.72 [95\% CI: 0.62 to 0.83 ]; $\mathrm{p}<0.001){ }^{25}$

Real-world outcomes of FOLFIRINOX versus gemcitabine plus nab-paclitaxel in patients with advanced pancreatic cancer were assessed in a population-based propensity scoreweighted analysis $(n=1,130) .{ }^{28}$ In this study, FOLFIRINOX, as compared with gemcitabine plus nab-paclitaxel, was associated with improved median OS, less frequent all-cause emergency department visits and all-cause hospitalizations but with an increased rate of febrile neutropenia-related hospitalization. Of note, the median OS was lower than in both the PRODIGE/ACCORD 11 study for FOLFIRINOX (8.2 months vs 11.1 months) and the MPACT study for nabpaclitaxel plus gemcitabine (6.1 months vs 8.7 months).

In Switzerland, a group of clinicians has built a consensus on the treatment strategy of metastatic PDAC in the first-line setting. ${ }^{23}$ The most used therapy is gemcitabine plus nabpaclitaxel, while in young (age below 65 years) and fit (ECOG PS of 0-1) patients with normal bilirubin levels, treatment with FOLFIRINOX can also be considered (Figure 1). As FOLFIRINOX provides a considerably higher ORR than gemcitabine plus nab-paclitaxel (30\% vs 20\%), this combination could be the preferred option in patients in whom tumor shrinkage represents the main clinical goal. Gemcitabine monotherapy remains an option for patients whose PS or comorbidities preclude combination chemotherapy. Finally, in frail patients with ECOG PS 3-4, best supportive care (BSC) is recommended. ${ }^{29}$

In order to optimize the continuum of care for patients with PDAC, first-line treatment choice should be based on the availability and overlap with potential second-line options. ${ }^{23}$ For example, there is no clinically validated secondline option after progression on frontline FOLFIRINOX, while gemcitabine plus nab-paclitaxel allow for second-line treatment with nanoliposomal irinotecan (nal-IRI) plus 5-fluorouracil and leucovorin (5-FU/LV), the only secondline therapy for metastatic pancreatic cancer that has shown a survival advantage in a phase III study, after progression to a gemcitabine-based regimen. ${ }^{30}$

\section{SECOND-LINE PALLIATIVE SETTING}

Approximately half of the patients with advanced pancreatic cancer ever reach second-line therapy. ${ }^{31}$ The selection of second-line therapy should be based on various factors such as drug availability, patient characteristics, physician preference, prior first-line therapy and the risk:benefit ratio. ${ }^{32}$

For the treatment of patients with metastatic PDAC whose disease progressed on gemcitabine plus nab-paclitaxel, nal-IRI in combination with 5-FU/LV is currently the only secondline therapy approved by Swissmedic in this clinical setting ${ }^{33}$ (Figure 1), based on the findings from the pivotal, phase III NAPOLI-1 trial ${ }^{34}$. In this study, 417 patients were randomly assigned to receive either nal-IRI plus 5-FU/LV $(n=117)$, nal-IRI monotherapy $(n=151)$ or 5 -FU/LV alone $(n=149){ }^{34}$ At data cutoff, nal-IRI in combination with 5-FU/LV versus 5-FU/LV alone significantly improved survival outcomes. The median OS was 6.1 months in patients nal-IRI plus 5-FU/LV and 4.2 months in those treated with 5-FU/LV only $(p=0.012)$. This survival benefit was sustained across key patient subgroups. When adjusted for prognostic factors, a multivariate analysis showed that nal-IRI plus 5-FU/LV remained associated with improved OS (HR: 0.58 [95\% CI: $0.42-0.81]$ ). In the final $O S$ analysis, the survival benefit of nal-IRI plus 5-FU/LV versus 5-FU/LV was sustained over an extended follow-up. ${ }^{35} \mathrm{Nal}-\mathrm{IRI}$ plus 5 -FU/LV also provided a PFS benefit, with a median PFS of 3.1 months, as compared with 1.5 months with 5-FU/LV alone (HR: 0.56 [95\% CI: $0.41-0.75] ; p=0.0001) .{ }^{34}$ In terms of safety, the most frequent grade 3-4 AEs with nal-IRI plus 5-FU/LV were neutropenia (27\%), fatigue (14\%) and diarrhea (13\%).

A post hoc sub-analysis of NAPOLI-1 further showed that increasing age is not prognostic of mortality or disease progression in PDAC patients treated with nal-IRI. ${ }^{36}$ Indeed, median OS and PFS were similar across all age subgroups indicating that treatment should not be withheld due to age alone in eligible and sufficiently fit patients. Further subgroup analyses identified other prognostic factors for worse clinical outcomes including decreased appetite at baseline, no prior curative surgery, presence of liver metastases, a greater number of distant metastases, and higher baseline pain and analgesic use. ${ }^{37,38}$ Based on the findings from NAPOLI-1, a predictive nomogram for OS at 6 and 12 months was developed. ${ }^{39}$ The univariate analyses identified 8 factors significantly associated with OS, including the Karnofsky PS, presence of liver metastasis, randomization to nal-IRI plus 5-FU/LV, albumin, neutrophil/lymphocyte ratio, CA 19-9, disease stage at diagnosis and body mass index. Despite several limitations of the study, the use of this nomogram may facilitate the decision-making process for gemcitabine-pretreated patients with PDAC.

In metastatic PDAC patients who progressed on a gemcitabinecontaining regimen, the combination of 5-FU plus oxaliplatin represents another treatment option. ${ }^{40}$ In a German phase III trial, a total of 168 patients were randomly assigned to receive 
either folinic acid and 5-FU (FF), or oxaliplatin plus FF (OFF). At a median follow-up of 54.1 months, the median OS was 5.9 months in the OFF group and 3.3 months in the FF group (HR: 0.66 [95\% CI: $0.48-0.91$ ]; $\mathrm{p}=0.010$ ). OFF was also associated with prolonged time to progression (2.9 months vs 2.0 months with FF; HR: 0.68 [95\% CI: 0.50-0.94]; $\mathrm{p}=0.019)$. Rates of AEs were generally similar between the two treatment arms, except for grade 1-2 neurotoxicity, which was more frequent among OFF-treated patients. However, the recent randomized, phase III PANCREOX trial showed no clinical benefit of 5-FU and oxaliplatin administered as modified FOLFOX6 (infusional 5-FU, LV, and oxaliplatin) versus infusional 5-FU in patients with advanced pancreatic cancer pretreated with gemcitabine-based therapy. ${ }^{41}$ Due to these contrasting results, the use of oxaliplatin in the second line remains controversial.

Real-world studies provided evidence that the effectiveness of nal-IRI may be consistent with clinical outcomes in the NAPOLI-1 trial, despite differences in patient characteristics and dosing patterns. ${ }^{42,43}$ In addition, our colleagues at the University Hospital Zurich in Switzerland, recently published a case report of a young, fit patient with metastatic PDAC and liver metastases, who demonstrated an outstanding clinical response to sequence treatment following the MPACT and NAPOLI-1 study protocols. ${ }^{44}$

Nal-IRI may also be considered for the treatment of patients with pancreatic cancer pretreated with irinotecan, especially those without disease progression during previous irinotecan treatment. A retrospective study presented at the ASCO Gastrointestinal (GI) Cancers Symposium 2021, which aimed to assess the potential role of nal-IRI in 64 patients who were pretreated with IRI, showed the median OS of 5.1 months from nal-IRI initiation. ${ }^{45}$ Furthermore, a post hoc analysis demonstrated that patients without previous progression on irinotecan had improved OS with nal-IRI, as compared with patients who had progressed on irinotecan (median, 6.1 months vs 4.3 months; $\mathrm{p}=0.0006$ ).

In the NAPOLI-1 trial, all patients underwent UGT1AI genotype testing. ${ }^{34}$ For 7 patients who were homozygous for the $U G T 1 A 1^{*} 28$ allele, the initial nal-IRI dose was reduced by $20 \mathrm{mg} / \mathrm{m}^{2}$ and then increased to the standard dose after the first cycle in the absence of drug-related toxic effects. Altogether, 5 patients were able to escalate to the standard starting dose, while one patient needed a further dose reduction, and one discontinued the treatment due to grade 3 vomiting. In Swiss clinics, genetic testing of UGTIAI polymorphisms prior to nal-IRI treatment is not routinely done. ${ }^{23}$ Although it is recommended, no clear guidelines regarding its applicability are available and as an option, it is possible to start at a lower dose level as a precaution, without affecting the overall efficacy. As an example, a recent post hoc analysis demonstrated that an early dose reduction or delay of liposomal irinotecan plus 5-FU/LV during the first 6 weeks of treatment does not significantly impact OS or PFS, as compared to patients without dose modifications. ${ }^{46}$

In the clinical setting, nal-IRI plus 5-FU/LV can also be considered as third-line therapy if previously not used (Figure 1).

After progression on frontline FOLFIRINOX, the combination of gemcitabine plus nab-paclitaxel may represent a second-line treatment option. This combination has been more effective than gemcitabine monotherapy in small retrospective and cohort studies. ${ }^{47-49}$

\section{BEYOND CHEMOTHERAPY}

About $15 \%$ of patients with pancreatic cancer have homologous recombination deficiency (HRD) such as germline mutations in the $B R C A 1 / 2$ genes. HRD results in impaired double-strand DNA break repair, leading to increased sensitivity to platinumbased chemotherapeutic regimens. Patients with HRD are also sensitive to poly-ADP ribose polymerase (PARP) inhibitors, which increase cytotoxicity by inhibiting the repair of singlestrand DNA breaks. ${ }^{50}$ As of now, several PARP inhibitors have been approved for the treatment of patients with $B R C A 1 / 2$ mutated advanced ovarian and breast cancers and are actively being studied for the management of patients with $B R C A 1 / 2$ mutated pancreatic adenocarcinoma. ${ }^{31}$

Olaparib, a PARP inhibitor, has been recently approved by Swissmedic as maintenance therapy in adult patients with metastatic adenocarcinoma of the pancreas with a germline $B R C A$ mutation whose disease has not progressed for a minimum of 16 weeks on first-line platinum-containing chemotherapy. ${ }^{51}$ This is based on the results of the phase III POLO trial, which assessed maintenance olaparib in patients with a germline $B R C A 1 / 2$ mutation and metastatic pancreatic cancer that had not progressed during first-line platinumbased chemotherapy. ${ }^{52}$ The median PFS was significantly longer in the olaparib arm than in the placebo arm (7.4 months vs 3.8 months; HR: 0.53 [95\% CI: 0.35-0.82]; $\mathrm{p}=0.004)$. At a data maturity of $46 \%$, no difference in OS between the two treatment groups was found. In terms of safety, the incidence of grade $\geq 3$ AEs was $40 \%$ with olaparib and $23 \%$ with placebo. There were no significant differences in health-related quality of life between the two arms.

Although rare (1\%), NTRK gene fusions are known to be oncogenic drivers in PDAC. Larotrectinib, an inhibitor of tropomyosin receptor kinases $\operatorname{Trk} A, \operatorname{TrkB}$ and $\operatorname{TrkC}$, demonstrated marked and durable antitumor activity in 159 adult and pediatric patients with NTRK fusion-positive cancer, regardless of tumor type. ${ }^{53,54}$ In a pooled analysis, the ORR according to investigator assessment was $79 \%$, including a complete response rate of $16 \% .^{54}$ In the safety population 
$(n=260)$, the most common grade 3-4 larotrectinib-related AEs were increased alanine aminotransferase (ALT) (3\%), anemia (2\%) and decreased neutrophil count (2\%). The most common larotrectinib-related serious AEs were increased ALT and aspartate aminotransferase (AST) and nausea (each $<1 \%)$. Larotrectinib was recently approved in Switzerland for use in patients with solid tumors that display an NTRK gene fusion. ${ }^{55}$

The majority of pancreatic cancer patients lack actionable mutations. ${ }^{56}$ To identify predictive biomarkers for optimal chemotherapy selection, this patient population was studied in the COMPASS trial. ${ }^{57}$ Fresh tumor samples underwent wholeexome and RNA sequencing to identify predictive mutational and transcriptional features for better chemotherapy selection. Results showed that prospective genomic profiling of advanced PDAC is feasible and that chemotherapy response differs among patients with different genomic/transcriptomic subtypes. For example, basal-like PDAC responds worse to chemotherapy, especially FOLFIRINOX, as compared with a classical subtype. Potentially actionable genetic alterations were found in $30 \%$ of patients.

In the PDAC setting, immunotherapy has demonstrated efficacy only in a subset of patients with microsatellite instability-high (MSI-H), which is present in $<1 \%$ of patients with pancreatic cancer. ${ }^{58}$ However, the phase II KEYNOTE 158 study, which investigated pembrolizumab in patients with noncolorectal MSI-H repair-deficient cancer, showed that PDAC patients achieved inferior response rates, when compared with patients with other types of cancer. ${ }^{59}$ More specifically, of 22 patients with pancreatic cancer, 1 had a complete response and 3 patients had a partial response. It is hypothesized that thelow antitumor activity of immunotherapy in PDAC patients stems from the low immunogenicity and the low tumor mutational burden. ${ }^{60}$ Furthermore, the abundant desmoplasmic stroma typical for PDAC generates a hypoxic microenvironment. This, in turn, drives the recruitment of
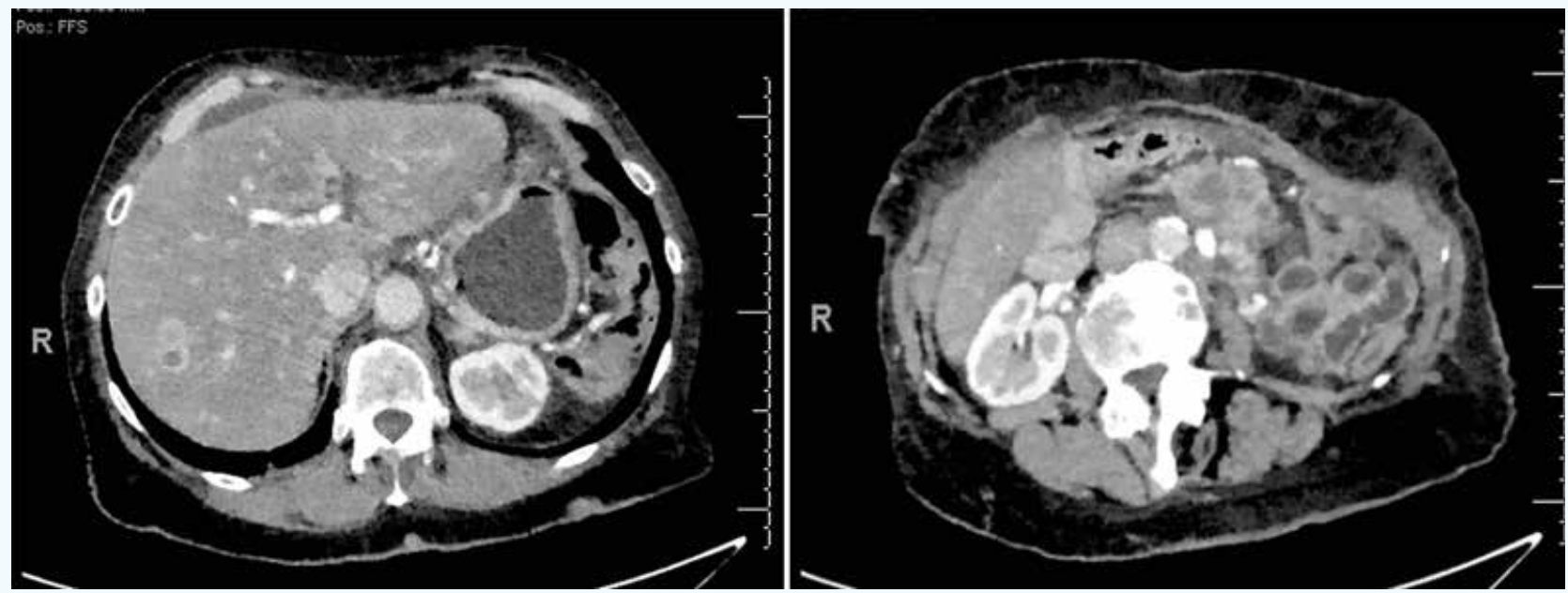

Figure 2. CT scan performed in July 2019, axial plane: Liver lesions in the right and left liver lobes and an abdominal mass in the pancreatic lodge. immunosuppressive cells through cancer-associated fibroblast activation and transforming growth factor (TGF) $\beta$ secretion, which is a negative prognostic factor. There are ongoing correlative studies that aimed to assess biomarkers that may predict immune sensitivity in this setting.

Although the KRAS mutation is the major oncogenic event in PDAC, it was considered undruggable until only recently. The KRAS G12C mutation occurred in about $3 \%$ of patients with PDAC. The phase I CodeBreaK100 trial investigated sotorasib, an oral KRAS G12C inhibitor, in heavily pretreated patients with KRAS p.G12C-mutant advanced non-small cell lung cancer (NSCLC), colorectal cancer and other solid tumors including pancreatic cancer. ${ }^{61}$ At data cutoff, 6 of 8 evaluable patients with pancreatic cancer achieved stable disease, of which 3 had a $30 \%$ reduction in tumor burden from baseline. These results show encouraging anticancer activity in heavily pretreated PDAC patients with KRAS G12C. Another novel agent targeting KRAS G12C is adagrasib (MRTX849), which also demonstrated encouraging efficacy in patients with advanced NSCLC, colorectal cancer and other types of tumors harboring this molecular alteration. ${ }^{62}$ In the phase I/ II KRYSTAL-1 study, 1 PDAC patient treated with adagrasib had a confirmed partial response.

Currently, molecular testing and upfront panel testing using next-generation sequencing (NGS) are used in Swiss clinics to identify potential oncogenic drivers. ${ }^{23}$ Genomic data generated by NGS may allow the development of personalized treatment programs with targeted therapies within clinical trials, given the large number of gene mutations seen in PDAC.

REAL-WORLD CLINICAL EXPERIENCE WITH NANOLIPOSOMAL IN SWITZERLAND

The favorable results of this treatment have been illustrated in two case reports. In the first one, as previously mentioned, a 48-year-old patient with metastatic pancreatic cancer 

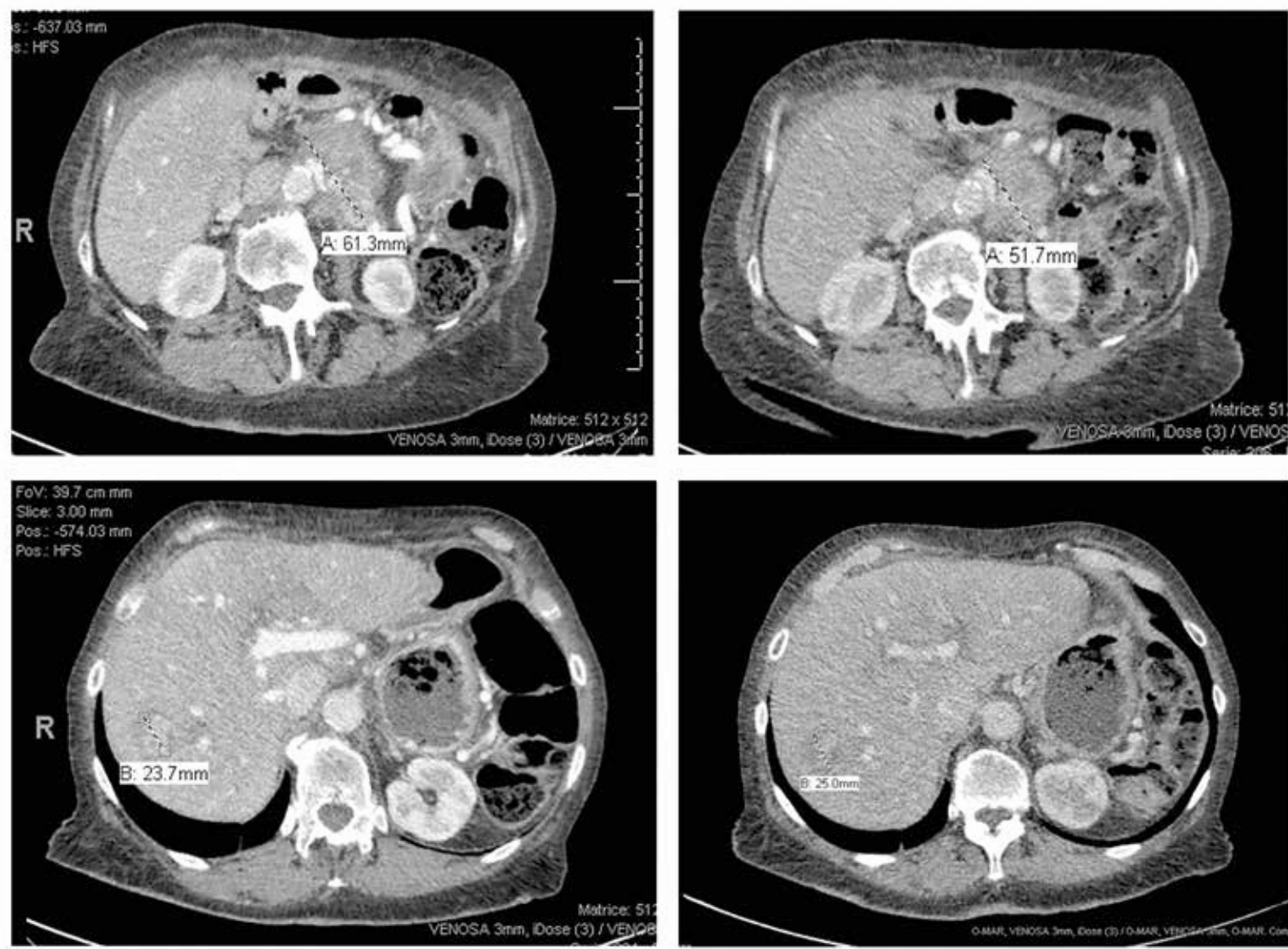

Figure 3. Local recurrence of the disease in August 2019, baseline CT scan prior to the treatment with nanoliposomal irinotecan (images on the left side). Disease stabilization according to the RECIST criteria in February 2020 (images on the right side).

demonstrated an exceptionally prolonged response to nal-IRI plus 5 -FU/LV in the third-line, following resistance to firstline FOLFIRINOX (dose-reduced) and second-line treatment with gemcitabine plus nab-paclitaxel leading to progression in the liver. ${ }^{44}$ Furthermore, the fact that irinotecan in the first-line failed to induce a good response, despite leading to impressive results in the third-line (as nal-IRI) might be explained by the fact that the mechanism of action of irinotecan is different between the two treatment schedules. This case further highlights the need for more accurate predictive biomarkers for the selection of optimal systemic treatment sequence.

Nal-IRI plus 5-FU also demonstrated a notably good response and safety profile in another case of a long-term survivor with a poorly differentiated advanced pancreatic cancer, as described below.

\section{CASE PRESENTATION}

A woman born in 1953 had spleno-pancreatectomy in February 2011 for grade 3 PDAC of $3.5 \mathrm{~cm}$ in diameter (pT3) with metastases in 5 out of 17 peripancreatic lymph nodes
(pN1) and no distant metastases (M0). After the surgery, the patient received adjuvant gemcitabine for 6 months.

In January 2018, 7 years from the initial diagnosis, the patient experienced local recurrence, for which she underwent duodeno-cefalo-pancreatectomy but was unable to receive adjuvant chemotherapy due to post-operative complications. She received supplementation with pancreatic enzymes, and she suffered from chronic diarrhea.

In June 2018, 6 months after the surgery, the patient completely recovered. At this time, a CT scan revealed another relapse in the abdominal lymph nodes and pancreatic lodge. Between July 2018 and August 2019, the patient was treated with first-line gemcitabine plus nab-paclitaxel, leading to a good response with acceptable toxicity. About 6 months after treatment initiation, the patient had short treatment holidays; thereafter, the treatment was restarted, and overall, the patient was treated for around one year. During this time, she experienced grade 2 sensitive neuropathy, which eventually decreased to grade 1 . 
At the end of July 2019, the patient experienced local disease progression with a mass in the pancreatic lodge as well as new liver lesions (Figure 2). In September 2019, the patient received second-line treatment with nal-IRI plus 5-FU/LV at a reduced dose of nal-IRI $\left(60 \mathrm{mg} / \mathrm{m}^{2}\right)$ due to a pancreatic insufficiency as a result of the radical pancreatectomy. Since the first cycle of therapy had been well-tolerated, the dose was increased to $80 \mathrm{mg} / \mathrm{m}^{2}$ in the second cycle, ultimately leading to disease stabilization as per the RECIST criteria. There were no significant adverse events except for steatorrhea, which was managed with dietary improvements and the addition of pancreatic enzymes. UGT1A1 testing was not performed. At the first evaluation in November 2019, disease stabilization was documented and confirmed in February 2020 (Figure 3 ). The treatment was carried on until June 2020, with good tolerability and overall disease control. At that time, a CT scan showed a slight enlargement of abdominal lymph nodes and initial signs of peritoneal carcinomatosis such as mild ascites and thickening and enhancement of peritoneal reflections. The patient's general condition quickly deteriorated accompanied by abdominal pain, diarrhea and vomiting. The treatment was interrupted and a palliative approach was set up. The patient died in September 2020.

\section{CONCLUSION}

- Due to a lack of specific symptoms and effective early detection methods, most patients with PDAC are diagnosed at advanced stages and are mainly treated palliatively.

- Despite significant treatment advances in the past years, the prognosis of patients with pancreatic cancer remains dismal.

- Chemotherapy is the mainstay of PDAC treatment, while novel targeted and immunomodulatory therapies are gradually expanding the treatment landscape.

- In Switzerland, the most used frontline therapy is gemcitabine plus nab-paclitaxel, while FOLFIRINOX can be considered in young and fit patients with a normal liver function, and gemcitabine monotherapy in frail patients.

- Molecular testing and upfront panel testing by next-generation sequencing (NGS) are used to identify the oncogenic driver.

- In Switzerland, genetic testing of UGT1A1 polymorphisms before treatment with nal-IRI is not routinely done. However, it is possible to start at a lower dose level as a precaution.

- The clinical use of nanoliposomal irinotecan (nal-IRI) in Switzerland is quite variable as it has been used both in the second- and third-line settings based on a clinical decision and previous lines of therapy.

\section{Declaration of Competing Interest}

Authors declare that they have no known competing financial interests or personal relationships that could have appeared to influence the work reported in this paper. 
REFERENCES

1. Kleeff J et al. Pancreatic cancer. Nat Rev Dis Primer. 2016; 2: 16022 .

2. Brunner $\mathrm{M}$ et al. Current Clinical Strategies of Pancreatic Cancer Treatment and Open Molecular Questions. Int J Mol Sci. 2019; 20(18): 4543

3. Bray F et al. Global cancer statistics 2018: GLOBOCAN estimates of incidence and mortality worldwide for 36 cancers in 185 countries. CA Cancer J Clin. 2018; 68(6): 394-424.

4. Ferlay $J$ et al. More deaths from pancreatic cancer than breast cancer in the EU by 2017. Acta Oncol. 2016; 55(910): $1158-60$.

5. Rawla P et al. Epidemiology of Pancreatic Cancer: Global Trends, Etiology and Risk Factors. World J Oncol. $2019 ; 10(1): 10-27$

6. Allemani $C$ et al. Global surveillance of trends in cancer survival 2000-14 (CONCORD-3): analysis of individual records for 37513025 patients diagnosed with one of 18 cancers from 322 population-based registries in 71 countries. Lancet. 2018; 391(10125): 1023-75.

7. National cancer statistic (NKS). BFS 2018. [Accessed May 2021]. Available from: https://wwwbfs.admin. $\mathrm{ch} / \mathrm{bfs} / \mathrm{en} / \mathrm{home} /$ statistics/health/surveys/ke.assetdetail.6706410.html.

8. Camcer. BFS 2020. [Accessed May 2021]. Available from: https://www.bfs.admin.ch/bfs/en/home/statistics/ from: https://www.bfs.admin.ch/bfs/en/

9. Siegel RL et al. Cancer statistics, 2019. CA Cancer J Clin. 2019; 69(1): 7-34.

2019; 69(1): 7-34.
10. Cancer Facts \& Figures 2020. American Cancer Society 10. Cancer Facts \& Figures 2020. American Cancer Society
2020. [Accessed May 2021]. Available from: https://www. cancer.org/research/cancer-facts-statistics/all-cancer-factsfigures/cancer-facts-figures-2020.html.

11. Montemurro $M$ et al. Trends in Pancreatic Cancer Survival in Switzerland. Schweiz Krebsbulletin Nr 42013. 2013; (4): 328-32.

12. Usón Junior PLS et al. The impact of metastatic sites in advanced pancreatic adenocarcinoma, systematic review and meta-analysis of prospective randomized studies. PLoS One. 2020; 15 (3): e0230060.

13. Swiss cancer report 2015: current situation and devel13. Swiss cancer report 2015: current situation and develhttps://www.bfs.admin.ch/bfs/en/home/statistics/health. https://www.bfs.admin.

14. Capasso $M$ et al. Epidemiology and risk factors of pancreatic cancer. Acta Biomed. 2018; 89(Suppl-9): 141-6.

15. McGuigan A et al. Pancreatic cancer: A review of clinical diagnosis, epidemiology, treatment and outcomes. World J Gastroenterol. 2018; 24(43): 4846-61.

16. Stjepanovic $\mathrm{N}$ et al. Hereditary gastrointestinal cancers: ESMO Clinical Practice Guidelines for diagnosis, treatment and follow-up ${ }^{\dagger}$. Ann Oncol. 2019; 30(10): 1558-71. 17. Sohal DPS et al. Metastatic Pancreatic Cancer: ASCO Clinical Practice Guideline Update. J Clin Oncol. 2018; 36(24): 2545-56.

18. Burris 3rd HA et al. Improvements in survival and clinical benefit with gemcitabine as first-line therapy for patients with advanced pancreas cancer: a randomized trial. J Clin with advanced pancreas cancer:
Oncol. 1997; 15(6): 2403-13

19. Gemcitabin Labatec $c^{5}$. Product information. Swissmedic 19. Gemcitabin Labatec. Product information. Swissmedic 2019. [Accesse
dicinfo.ch.

20. Oettle $\mathrm{H}$ et al. Adjuvant chemotherapy with gemcitabine and long-term outcomes among patients with resected pancreatic cancer: the CONKO-001 randomized trial. JAMA. 2013; 310(14): 1473-81.

21. eUpdate - Cancer of the Pancreas Treatment Recommendations. ESMO Guidelines Committee. [Accessed May 2021]. Available from: https://www.esmo.org/guidelines/gastrointestinal-cancers/pancreatic-cancer/eupdatecancer-of-the-pancreas-treatment-recommendations.

22. Conroy T et al. FOLFIRINOX versus gemcitabine for metastatic pancreatic cancer. N Engl J Med. 2011;364(19): $1817-25$.
23. De Dosso $S$ et al. Treatment landscape of metastatic pancreatic cancer. Cancer Treat Rev. 2021;96: 102180. 24. Von Hoff DD et al. Increased Survival in Pancreatic Cancer with nab-Paclitaxel plus Gemcitabine. N Engl Med. 2013;369(18): 1691-703.

25. Goldstein D et al. nab-Paclitaxel Plus Gemcitabine for Metastatic Pancreatic Cancer: Long-Term Survival From Phase III Trial. J Natl Cancer Inst. 2015; 107(2): dju413. 26. Tabernero J et al nab-Paclitaxel plus gemcitabine for metastatic pancreatic nancer: a subroup analysis of Western European Western European cohort of

27. Scheithauer W et al. Dose modification and efficacy of nab-paclitaxel plus gemcitabine vs. gemcitabine for patients with metastatic pancreatic cancer: phase III MPACT trial Gastrointest Oncol. 2016; 7(3): 469-78.

28. Chan KKW et al. Real-world outcomes of FOLFIRINOX vs gemcitabine and nab-paclitaxel in advanced pancreatic cancer: A population-based propensity scoreweighted analysis. Cancer Med. 2019; 9(1): 160-9.

29. Glatzer $\mathrm{M}$ et al. Choice of first line systemic treatment in pancreatic cancer among national experts: Subgroup Analyses of Patient, Tumor, and Previous Treatment Characteristics in the Pivotal NAPOLI-1 Trial. Pancreatology. 2020; 20(4): 686-90.

30. Macarulla Mercadé $T$ et al. Liposomal Irinotecan + 5-FU/LV in Metastatic Pancreatic Cancer. Pancreas. 2020; 49(1): 62-75.

31. Lambert $A$ et al. An update on treatment options for pancreatic adenocarcinoma. Ther Adv Med Oncol. 2019; 11 25568.

32. Taieb J et al. First-line and second-line treatment of patients with metastatic pancreatic adenocarcinoma in routin clinical practice across Europe: a retrospective, observation chart review study. ESMO Open. 2020; 5(1): e000587.

33. Onivyde (irinotecan). Product information. Swissmedic 2019. [Accessed May 2021]. Available from: www.swissmedicinfo.ch

34. Wang-Gillam A et al. Nanoliposomal irinotecan with fluorouracil and folinic acid in metastatic pancreatic cancer after previous gemcitabine-based therapy (NAPOLI-1): a global, randomised, open-label, phase 3 trial. Lancet. 2016; 387(10018): 545-57.

35. Wang-Gillam A et al. NAPOLI-1 phase 3 study of liposomal irinotecan in metastatic pancreatic cancer: Final overall survival analysis and characteristics of long-term survivors. Eur J Cancer. 2019; 108: 78-87.

36. Mercadé TM et al. Liposomal irinotecan and 5-fluorouracil/leucovorin in older patients with metastatic pancreatic cancer - A subgroup analysis of the pivotal NAPOLI-1 trial. J Geriatr Oncol. 2019; 10(3): 427-35.

37. Mercadé TM et al. Selected subgroup analyses of liposomal irinotecan (nal-IRI) in patients with metastatic pancretic ductal adenocarcinoma (mPDAC) in the global NAPOLI-1 phase III trial. Ann Oncol. 2018; 29(Suppl-5): v101.

38. Mercadé TM et al. Liposomal Irinotecan + 5-FU/LV in Meraste Pancteac Casor: Subgroup Analyses of $\mathrm{Pa}^{-}$ tient, Tumor, and Previous tient, Thor Piv. Che (1): 62-75. 39. Chen L-Tet aredicting Survival in Patients Treated with Liposomal Irinotecan Plus Fluorour-
acil and Leucovorin in Metastatic Pancreatic Cancer. Canacil and Leucovorin in Metastatic
cers (Basel). 2019; 11(8): 1068.

cers (Basel). 2019; 11(8): 1068 .
40. Oettle $\mathrm{H}$ et al. Second-line oxaliplatin, folinic acid, 40. Oettle $\mathrm{H}$ et al. Second-line oxaliplatin, folinic acid and fluorouracil versus folinic acid and fluorouracil alone for gemcitabine-refractory pancreatic cancer: outcomes from the

41. Gill $S$ et al. PANCREOX: A Randomized Phase III Study of Fluorouracil/Leucovorin With or Without Oxaliplatin for Second-Line Advanced Pancreatic Cancer in Patients Who Have Received Gemcitabine-Based Chemotherapy. J Clin Oncol. 2016; 34(32): 3914-20.

42. Barzi A et al. Real-World Dosing Patterns and Outco42. Barzi A et al. Real-World Dosing Patterns and Outco-
mes of Patients With Metastatic Pancreatic Cancer Treated
With a Liposomal Irinotecan Regimen in the United States. Pancreas. 2020; 49(2): 193-200.

43. Kieler $\mathrm{M}$ et al. A real-world analysis of second-line treatment options in pancreatic cancer: liposomal-irinotecan
plus 5 -fluorouracil and folinic acid. Ther Adv Med Oncol 2019; 11: 1758835919853196.

44. Akhoundova Sanoyan D et al. Sequential Treatment of Metastatic Adenocarcinoma of the Pancreatic Duct with Liver Metastasis Following the NAPOLI-1 Study Protocol with nal-Irinotecan plus 5-FU in the Second Line. Case Reps Oncol. 2020; 13(1): 79-84.

45. Smith CJ et al. Nanoliposomal irinotecan-based chemotherapy after regular irinotecan-based chemotherapy in patients with pancreas cancer. ASCO Gastrointestinal Cancers Symposium; 15-17 January 2021. Poster presentation 402

46. Chen L-T et al. Early dose reduction/delay and the efficacy of liposomal irinotecan with fluorouracil and leucovorin in metastatic pancreatic ductal adenocarcinom (mPDAC): A post hoc analysis of NAPOLI-1. Pancreatology. 2021; 21(1): 192-9.

47. Portal A et al. Nab-paclitaxel plus gemcitabine for metastatic pancreatic adenocarcinoma after Folfirinox failure: an 113(7): 989-95.

48. de Jesus VHF et al Systematic review and meta-analysis of gemcitabine-based chemotherapy after FOLFIRINOX of gemcitabine-based chemotherapy after FOLFIRINOX in advanced pancreatic cance 1758835920905408 .

49. de Jesus VHF et al. Retrospective analysis of efficacy and safety of Gemcitabine-based chemotherapy in patients with metastatic pancreatic adenocarcinoma experiencing disea-
se progression on FOLFIRINOX. I Gastrointest Oncol. se progression on $\mathrm{F}$

50. Lord CJ, Ashworth A. The DNA damage response an cancer therapy. Nature. 2012; 481(7381): 287-94.

51. Lynparza (olaparib). Product information. Swissmedic 2020. [Accessed May 2021]. Available from: www.swissmedicinfo.ch

52. Golan T et al. Maintenance Olaparib for Germline BRCA -Mutated Metastatic Pancreatic Cancer. N Engl J Med. 2019; 381(4): 317-27.

53. Drilon A et al. Efficacy of Larotrectinib in TRK Fusion-Positive Cancers in Adults and Children. N Engl J Med. 2018; 378(8): 731-9.

54. Hong DS et al. Larotrectinib in patients with TRK fusion-positive solid tumours: a pooled analysis of three phas 1/2 clinical trials. Lancet Oncol. 2020; 21(4): 531-40. 55. Vitrakvi (larotrectinib). Swissmedic 2020. Product information. [Accessed April 2021]. Available from: www. swissmedicinfo.ch.

56. Akhoundova D et al. Precision Oncology for Hepato-Pancreato-Biliary (HPB) Cancers: State of the Art and Future Directions. healthbook TIMES Onco Hema 2020 (5): 52-59..

57. Aung KL et al. Genomics-Driven Precision Medicine for Advanced Pancreatic Cancer: Early Results from the COMPASS Trial. Clin Cancer Res. 2018; 24(6): 1344-54. 58. Le DT et al. Mismatch repair deficiency predicts response of solid tumors to PD-1 blockade. Science. 2017; ponse of solid tumors

59. Marabelle A et al. Efficacy of Pembrolizumab in Patients With Noncolorectal High Microsatellite Instability/Mismatch Repair-Deficient Cancer: Results From the Phase I KEYNOTE-158 Study. J Clin Oncol. 2020; 38(1): 1-10. 60. Hilmi $\mathrm{M}$ et al. Immune therapies in pancreatic ducta adenocarcinoma: Where are we now?. World J Gastroenterol. 2018; 24(20): 2137-51.

61. Hong DS et al. KRASG12C Inhibition with Sotorasib in Advanced Solid Tumors. N Engl J Med. 2020; 383(13): $1207-17$

62. Johnson ML et al. KRYSTAL-1: Activity and Safety of Adagrasib (MRTX849) in Patients with Colorectal Cancer (CRC) and Other Solid Tumors Harboring a KRAS G12C Mutation. European Journal of Cancer. 2020; 138: S2 